\title{
Erratum to: Understanding Kappa Distributions: A Toolbox for Space Science and Astrophysics
}

\section{G. Livadiotis · D.J. McComas}

Published online: 1 June 2013

(C) Springer Science+Business Media Dordrecht 2013

\section{Erratum to: Space Sci Rev \\ DOI 10.1007/s11214-013-9982-9}

Unfortunately, a mistake was introduced in Eq. (3.16) during the typesetting process of this article. Please find the correct equation below:

$$
P(\boldsymbol{u} ; \theta, \kappa) \cong \pi^{-\frac{3}{2}} \frac{\Gamma(\kappa+1)}{\Gamma\left(\kappa-\frac{1}{2}\right)}\left[\left(\kappa-\frac{3}{2}\right) \theta^{2}\right]^{\kappa-\frac{1}{2}} \cdot|\boldsymbol{u}|^{-2 \kappa-2} .
$$

The online version of the original article can be found under doi:10.1007/s11214-013-9982-9.

G. Livadiotis $(\bowtie) \cdot$ D.J. McComas

Southwest Research Institute, San Antonio, TX, USA

e-mail: glivadiotis@swri.edu

D.J. McComas

Department of Physics \& Astronomy, University of Texas at San Antonio, San Antonio, TX, USA 